\title{
Trachys deccanensis (Poaceae): A new species from semiarid zone of peninsular India
}

\author{
M. Anil Kumar, K.V. Subbaiah and B. Ravi Prasad Rao* \\ Biodiversity Conservation Division, Department of Botany, Sri Krishnadevaraya University, \\ Anantapuramu - 515003, Andhra Pradesh, India. \\ *E-mail: biodiversityravi@gmail.com
}

\begin{abstract}
Trachys deccanensis M. Anil Kumar \& B.R.P. Rao, a new species belongs to the tribe Paniceae, subfamily Panicoideae and family Poaceae is described here. The novel species is allied to T. narasimhanii Ravich. in having longer spikes, lanceolate spikelets and absence of vestigial floral glumes, but distinctly differs in leaf texture, spike arrangement, nature and number of spikelets in individual clusters at each node of the rachis. The new species is also allied to $T$. copeana Kabeer \& V.J. Nair in having two or three digitate racemes, single cluster of spikelets, number of spikelets in individual cluster at each node of the rachis; but distinctly differs in leaf texture, nature and texture of spikelets in individual cluster at each node of rachis and lanceolate spikelets. Description, illustration, microscopic images, comparative morphology of the new species with the allied species and a key for identification of Trachys species have been provided.
\end{abstract}

Keywords: Andhra Pradesh, India, New Grass, Trachys deccanensis

\section{Introduction}

Trachys Pers. was erected as a monospecific genus in the tribe Paniceae, of subfamily Panicoideae and family Poaceae with type species, T. muricata (L.) Pers. (Clayton \& Renvoize, 1986). Later, T. copeana Kabeer \& V.J. Nair (Kabeer \& Nair, 2009), T. narasimhanii Ravich. (Ravichandran, 2013) were added to the genus. All the three species of Trachys have been confined to India, Myanmar and Sri Lanka (Bor, 1960; Karthikeyan et al., 1989; Clayton et al., 2006; Kabeer \& Nair, 2007, 2009; Ravichandran, 2013); of these, T. muricata is found in India, Myanmar and Sri Lanka; T. copeana and T. narasimhanii are endemic to the state of Tamil Nadu, India. The genus is characterized by stoloniferous habit, tomentose leaves, 1-3 spike-like racemes, fragile leafy rachis, clusters of spikelets disarticulating along with rachis as segments (Clayton \& Renvoize, 1986; Watson \& Dallwitz, 1994).

Part of explorations held in 2016 in the semiarid zone of peninsular India, we could collect few curious specimens of grasses resembling Trachys. in the cultivated fields of Kadivella village near Yemmiganur, Kurnool district, Andhra Pradesh. Critical examination of pertinent literature and type specimens of all three species of Trachys namely $T$. copeana and T. narasimhanii deposited in Madras Herbarium and T. muricata through online Kew Herbarium Catalogue revealed that the present collection is allied to T. narasimhanii Ravich. and T. copeana Kabeer \& V.J. Nair, but distinctly differs in both vegetative and reproductive characters, which warrants new species status. The present paper provides description, illustration, digital microscopic images, and comparative morphology of the novel species with its allied species, $T$. narasimhanii and T. copeana (Table 1), and key for identification of Trachys species.

Trachys deccanensis M. Anil Kumar \& B.R.P. Rao, sp. nov.

Figs. 1,2

Trachys deccanensis is allied to $T$. narasimhanii Ravich., but distinctly differs from the latter in having glabrous leaves (densely tomentose in $T$. narasimhanii), digitate spikes (divaricate in $T$. narasimhanii), spikelets dissimilar in texture, sessile spikelets glabrous, pedicelled ones ciliate on lower lemmas (all spikelets are glabrous in T. narasimhanii), single cluster at each node comprising three spikelets (two clusters, each with two to seven spikelets in $T$. narasimhanii) and without any sterile spikelets (sterile spikelets present in T. narasimhanii). This species is also allied to T. copeana Kabeer \& V.J. Nair but 
Table 1. Comparison of Trachys deccanensis, T. copenana and T. narasimhanii

\begin{tabular}{|c|c|c|c|}
\hline Characters & T. deccanensis, sp. nov. & $\begin{array}{l}\text { T. copeana (based on } \\
\text { protologue) }\end{array}$ & $\begin{array}{l}\text { T. narasimhanii (based } \\
\text { on protologue) }\end{array}$ \\
\hline Culms & Up to $80 \mathrm{~cm}$ high & Up to $40 \mathrm{~cm}$ high & Up to $70 \mathrm{~cm}$ high \\
\hline Ligule & $\begin{array}{l}\text { 1-2 mm long, } \\
\text { membranous }\end{array}$ & $\begin{array}{l}0.6-0.8 \mathrm{~mm} \text { long, } \\
\text { membranous }\end{array}$ & $\begin{array}{l}1-2.5 \mathrm{~mm} \text { long, } \\
\text { tuberculate }\end{array}$ \\
\hline Leaf sheath & $\begin{array}{l}\text { Glabrous/nearly } \\
\text { glabrous }\end{array}$ & Pubescent & Pubescent \\
\hline Racemes & 3, digitate, $6-9 \mathrm{~cm}$ long & $\begin{array}{l}2 \text { or rarely } 3 \text {, digitate, up } \\
\text { to } 5.5 \mathrm{~cm} \text { long, }\end{array}$ & 2, divaricate, $2-9 \mathrm{~cm}$ long \\
\hline Peduncles & $\begin{array}{l}\text { 6-12 cm long, bearded } \\
\text { near apex }\end{array}$ & $\begin{array}{l}5-8 \mathrm{~cm} \text { long, bearded } \\
\text { near apex }\end{array}$ & $\begin{array}{l}\text { 5-11 cm long, bearded } \\
\text { near apex }\end{array}$ \\
\hline Spikelet clusters & $\begin{array}{l}\text { 5-6 mm apart, } \\
\text { comprising one cluster } \\
\text { of spikelets, each } \\
\text { cluster with } 3 \text { bisexual } \\
\text { spikelets, similar in size, } \\
\text { dissimialar in texture }\end{array}$ & $\begin{array}{l}4-5.5 \text { mm apart, } \\
\text { comprising one cluster of } \\
\text { spikelets, with } 3 \text { similar } \\
\text { bisexual spikelets }\end{array}$ & $\begin{array}{l}2-4 \text { mm apart, } \\
\text { comprising } 2 \text { clusters, } \\
\text { each cluster with } 2-7 \\
\text { spikelets of dissimilar in } \\
\text { size, often bisexual and } \\
\text { one spikelet is barren }\end{array}$ \\
\hline Sterile spikelets & Absent & Absent & Present \\
\hline Spikelets & $\begin{array}{l}\text { Lanceolate, } 5.3-5.6 \times \\
2-3 \mathrm{~mm} \text {, apex acute to } \\
\text { acuminate }\end{array}$ & $\begin{array}{l}\text { Broadly ovate, } 4-4.2 \times \\
2-3 \mathrm{~mm} \text {, apex acute }\end{array}$ & $\begin{array}{l}\text { Narrowly lanceolate, } 4-5 \\
\times 2-4 \mathrm{~mm} \text {, apex acute to } \\
\text { acuminate }\end{array}$ \\
\hline Pedicels & $1-1.5 \mathrm{~mm}$ long & $0.6-1 \mathrm{~mm}$ long & 1-2 mm long \\
\hline Lower Glume & $\begin{array}{l}0.2-0.5 \mathrm{~mm} \text { long, apex } \\
\text { obtuse }\end{array}$ & $\begin{array}{l}0.3-0.4 \mathrm{~mm} \text { long, apex } \\
\text { acute }\end{array}$ & $\begin{array}{l}0.2-0.5 \mathrm{~mm} \text { long, apex } \\
\text { obtuse }\end{array}$ \\
\hline Upper Glume & $\begin{array}{l}\text { 2-3.7 mm long, apex } \\
\text { acuminate, 3-veined, } \\
\text { sparsely ciliate at } \\
\text { margins or glabrous }\end{array}$ & $\begin{array}{l}\text { 1.8-3 mm long, acute } \\
\text { to acuminate, } 3 \text {-veined, } \\
\text { sparsely ciliate at } \\
\text { margins or glabrous }\end{array}$ & $\begin{array}{l}\text { 2-3.7 mm long, apex } \\
\text { acuminate, chartaceous, } \\
\text { 3-veined, sparsely ciliate } \\
\text { all along }\end{array}$ \\
\hline Lower Lemma & $\begin{array}{l}\text { Lanceolate, 3.5-5.5 } \\
\text { mm long, margins } \\
\text { thick, apex acuminate, } \\
\text { inconspicously } \\
\text { 9-11-veined }\end{array}$ & $\begin{array}{l}\text { Broadly ovate or } \\
\text { lanceolate, } 3.5-4.2 \mathrm{~mm} \\
\text { long, more or less bulged } \\
\text { and dome-shaped up to } \\
\text { middle and flat above, } \\
\text { margins hyaline, apex } \\
\text { acute, prominently } \\
\text { 11-13-veined (faint when } \\
\text { young) }\end{array}$ & $\begin{array}{l}\text { Broadly lanceolate, } \\
3.5-5 \mathrm{~mm} \text { long, margins } \\
\text { hyaline, apex acuminate, } \\
\text { coriaceous, } 9-11 \text {-veined }\end{array}$ \\
\hline Lower Palea & $\begin{array}{l}0.3-0.5 \mathrm{~mm} \text { long, often } \\
\text { reduced }\end{array}$ & $\begin{array}{l}0.7-1.4 \mathrm{~mm} \text { long, at times } \\
\text { reduced }\end{array}$ & $0.3-0.5 \mathrm{~mm}$ long, reduced \\
\hline Upper Lemma & $\begin{array}{l}\text { Lanceolate, 3-3.2 } \\
\text { mm long, acuminate, } \\
\text { 3-veined, hyaline }\end{array}$ & $\begin{array}{l}\text { Ovate to lanceolate, } \\
\text { 3.2-3.8 mm long, acute, } \\
\text { 3-veined, chartaceous }\end{array}$ & $\begin{array}{l}\text { Lanceolate, 3-3.2 } \\
\text { mm long, acuminate, } \\
\text { 3-veined, hyaline }\end{array}$ \\
\hline
\end{tabular}




\begin{tabular}{llll}
\hline Characters & T. deccanensis, sp. nov. & $\begin{array}{l}\text { T. copeana (based on } \\
\text { protologue) }\end{array}$ & $\begin{array}{l}\text { T. narasimhanii (based } \\
\text { on protologue) }\end{array}$ \\
\hline Upper Palea & $\begin{array}{l}\text { Lanceolate, 3-3.1 } \mathrm{mm} \\
\text { long, apex sharply acute, } \\
\text { thin-chartaceous }\end{array}$ & $\begin{array}{l}\text { Ovate to lanceolate, } \\
3.2-3.5 \mathrm{~mm} \text { long, apex } \\
\text { acute, chartaceous }\end{array}$ & $\begin{array}{l}\text { Lanceolate, } 3-3.1 \mathrm{~mm} \\
\text { long, apex acute, slightly } \\
\text { chartaceous }\end{array}$ \\
$\begin{array}{l}\text { Pedicelled } \\
\text { Spikelets }\end{array}$ & $\begin{array}{l}\text { Distinctly ciliate on } \\
\text { dorasal side of its sterile } \\
\text { lemmas }\end{array}$ & Glabrous & \\
Anthers & $1-1.2 \mathrm{~mm}$ long & $1.3-1.5 \mathrm{~mm}$ long & $1-1.5 \mathrm{~mm}$ long \\
\hline
\end{tabular}

distinctly differs in glabrous leaves (densely tomentose in $T$ copeana), spikelets dissimilar in texture, sessile spikelets lanceolate (ovate in $T$. copeana), glabrous and pedicelled ones ciliate on lower lemmas (all spikelets are glabrous in T. copeana).

Type: INDIA, Andhra Pradesh: Kurnool district, Cultivated fields of Kadivella village near Yemmiganur, 16.11.2016. B. Ravi Prasad Rao, K.V. Subbaiah \& M. Anil Kumar 52099 (holo, SKU; iso, CAL).

Stoloniferous annual or short-lived perennial. Culms to $80 \mathrm{~cm}$ long; nodes hairy, rooting from lower nodes. Leaves elliptic to linear-lanceolate, 4-11 $\times$ c. $0.8 \mathrm{~cm}$, obtuse to rounded, margins undulate, apex sharply acute to acuminate, flat, glabrous; ligules 1-2 mm long, hyaline, membranous; leaf sheath laterally compressed, sharply keeled in lower leaves, 3-10 cm long, enclose up to half of internode length, glabrous, margins thin-hyaline, mouth with few tuberculate hairs or glabrous. Racemes spike-like, three in digitate, 6-9 cm long, spikelets in clusters, arranged alternately on the under surface of rachis without any vestigial structures, clusters of spikelets arranged apart 4.5-6 $\mathrm{mm}$ distance, pubescent at the divergence zone; joints triquetrous, $4.5-6 \times 1.2-2.2 \mathrm{~mm}$, straight to curved, margins winged, glabrous, green, manyveined; peduncles 6-12 cm long, terete, bearded near apex. Raceme joints bulged, $2-3 \mathrm{~mm}$ across, comprising one cluster of spikelets; cluster size $6.5-7 \times 2-4 \mathrm{~mm}$, each cluster with three bisexual spikelets which are similar in size, dissimilar in texture; entire cluster disarticulating at maturity. Spikelets narrowly lanceolate, 5.3-5.6 × 2-3 mm, base truncate, apex acute-acuminate, coriaceous, yellow-brown; pedicels stout, 1-1.5 mm, greenish yellow. Glumes shorter than spikelets; lower glume reduced, scale-like, $0.2-0.5 \times 0.2-0.3 \mathrm{~mm}$, base truncate, apex obtuse, subcoriaceous, veinless.
Upper glume lanceolate, $2-3.7 \times 0.5-0.8 \mathrm{~mm}$, base truncate, apex obtuse, chartaceous, glabrous or sparsely ciliate at margins, green, prominently 3 -veined. Spikelets with 2 florets; lower barren with 2 lodicules; upper bisexual. Lower sterile lemma lanceolate, 3.5-5.5 × 2-3 mm, base truncate, apex obtuse, coriaceous, yellow-brown, inconspicuously 9-11-veined, margins thick, in-rolled. Lower palea oblong, often reduced, $0.3-0.5 \times 0.2-0.3 \mathrm{~mm}$. Upper lemma lanceolate, 3-3.2 × 1-2 mm, base truncate, apex obtuse, hyaline, 3-veined, margins in-rolled, envelop the margins of palea. Upper palea lanceolate, similar to its lemma in shape, 3-3.1 $\times$ 1-1.5 mm, thin-chartaceous. Lodicules 2. Stamens 3; anthers 1-1.2 mm long, yellow. Ovary oblong, $c$. $0.7 \times 0.3 \mathrm{~mm}$; style c. $2 \mathrm{~mm}$ long; stigma plumose, 1.5-2 mm long, light brown. Caryopsis not seen. Pedicelled spikelets similar to sessile spikelets in size and sex, but are hairy, hairs from dorsal side of lower sterile lemmas, usually in tow prominent lines in the middle.

\section{Flowering \& fruiting: June-December.}

Habitat: Rare, found in rainfed cultivated fields associated with Trachys muricata, Alloteropsis cimicina (L.) Stapf and Digitaria spp.

Distribution: Till date, this new species is known only from its type locality, Kadivella village near Yemmiganur, Kurnool district, Andhra Pradesh.

Etymology: The specific epithet is named after "Deccan Plateau", one of the biogeographic zones of India, where the type locality is situated.

Conservation Status: Seen only a few specimens in one occasion in the type locality. Further explorations are needed in adjacent similar habitats to estimate the exact size of the population and evaluate the conservation status of this species. However, its provisionally assessed here as Data Deficient. 


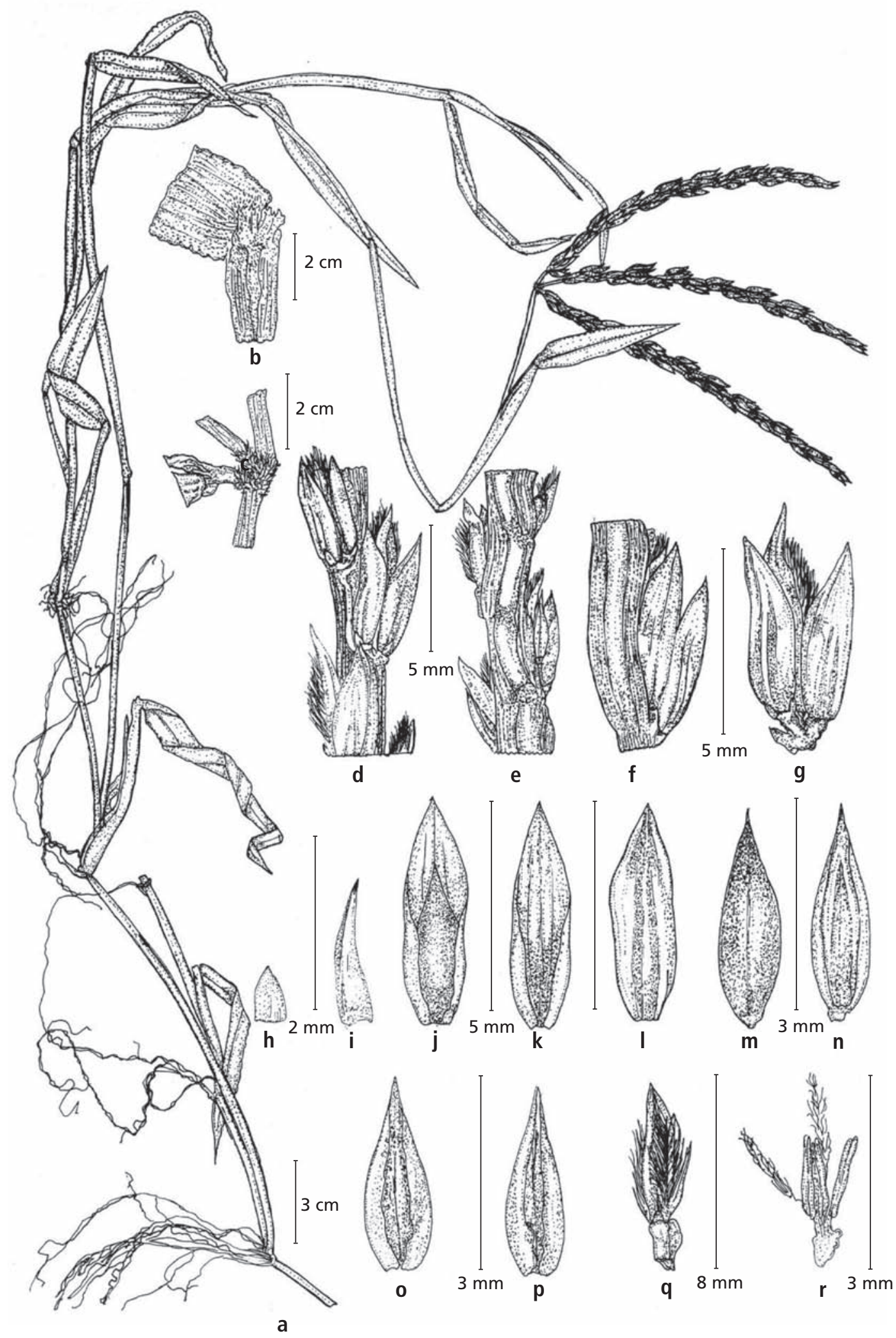

Fig. 1. Trachys deccanensis M. Anil Kumar \& B.R.P. Rao: a. Habit; b. Portion of leaf showing ligule; c. Divergent area of spikes; d. Portion of spike, ventral view; e. Portion of spike, dorsal view; f. Spikelets cluster, with leafy rachis; g. Cluster of spikelets without rachis; h. Lower glume; i. Upper glume; j. Florets of sessile spikelet; k. Sterile lemma of sessile spikelet, ventral view; I. Sterile lemma of sessile spikelet, dorsal view; m. Upper floret, dorsal view; $n$. Upper floret, ventral view; o. Lemma of fertile floret; $p$. Palea of fertile floret; q. Pedicelled spikelet; r. Stamens and Ovary. 


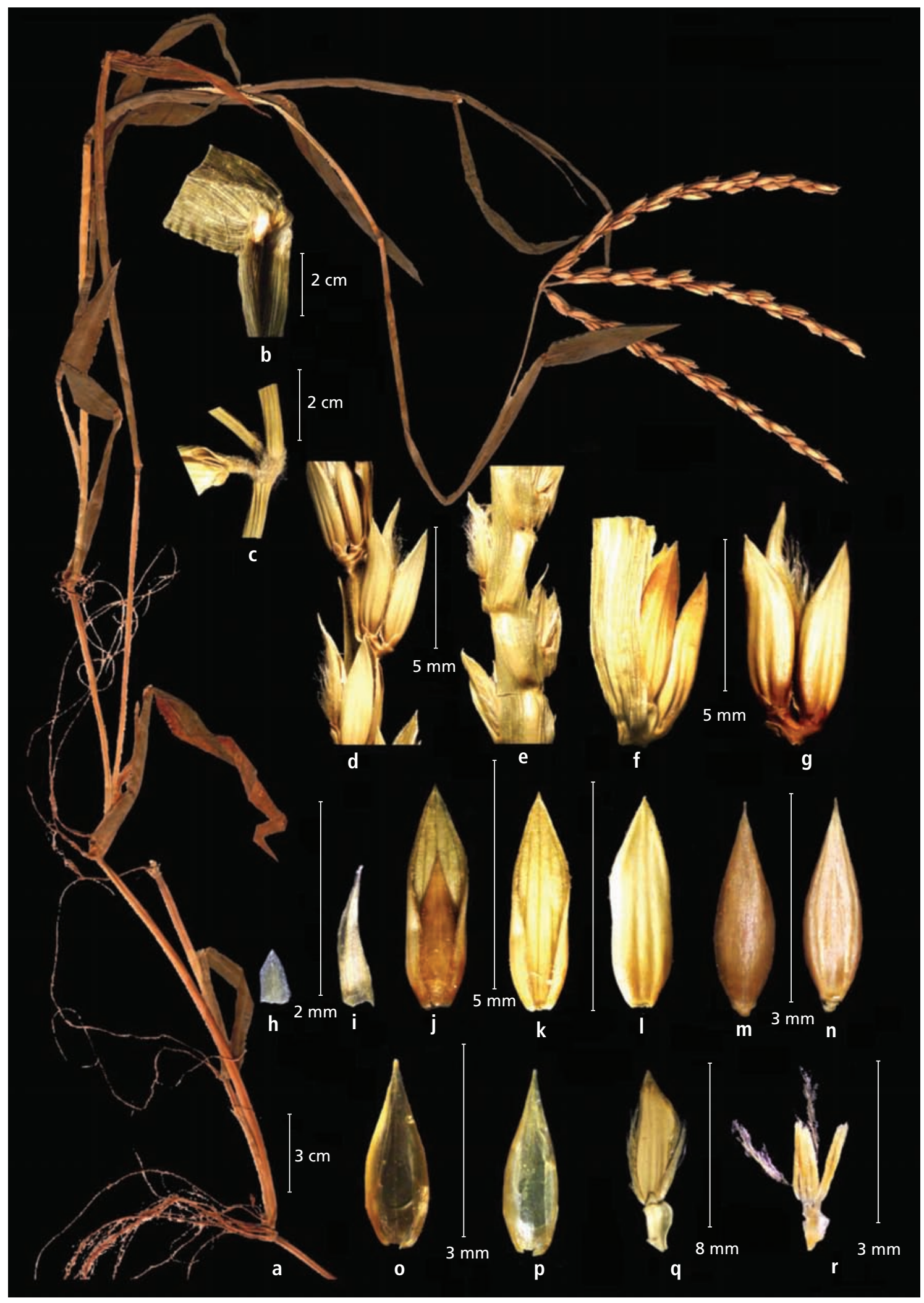

Fig. 2. Trachys deccanensis M. Anil Kumar \& B.R.P. Rao: a. Habit; b. Portion of leaf showing ligule; c. Divergent area of spikes; d. Portion of spike, ventral view; e. Portion of spike, dorsal view; f. Spikelets cluster with leafy rachis; g. Cluster of spikelets without rachis; h. Lower glume; i. Upper glume; j. Florets of sessile spikelet; k. Sterile lemma of sessile spikelet, ventral view; I. Sterile lemma of sessile spikelet, dorsal view; m. Upper floret, dorsal view; $n$. Upper floret, ventral view; o. Lemma of fertile floret; p. Palea of fertile floret; q. Pedicelled spikelet; r. Stamens and Ovary. 


\section{Key to the species of Trachys}

1. Clusters of spiketlets 2 or 3 at each rachis internodes

1. Clusters of spikelets single at each rachis internodes 3

2. Clusters of spikelets 3; sterile lemmas broadly ovate T. muricata

2. Clusters of spikelets 2; sterile lemmas lanceolate T. narasimhanii

3. Leaves tomentose; all spikelets glabrous T. copeana

3. Leaves glabrous; pedicelled spikelets hairy on lower lemmas T. deccanensis

\section{Acknowledgements}

BRPR gratefully acknowledges University Grants Commission, for BSR-One Time Grant Project (No. F. 19-151/2015 (BSR)). The first author grateful to National Remote Sensing Centre, Hyderabad, for providing the Junior Research Fellowship (NRSC/ FEG/VCP 2015).

\section{Literature Cited}

Bor, N.L. 1960. Grasses of Burma, Ceylon, India and Pakistan (excluding Bambuseae). Pergamon Press, Oxford.
Clayton, W.D. \& S.A. Renvoize 1986. Genera Graminum. Grasses of the World. Her Majesty's Stationery Office, London.

Clayton, W.D., Vorontsova, M.S., Harman, K.T. \& H. Williamson 2006 onwards. GrassBase - The Online World Grass Flora. http://www.kew. org/data/grass.db.html (Accessed: 1st March 2017)

Karthikeyan, S., Jain, S.K., Nayar, M.P. \& M. Sanjappa 1989. Florae Indicae Enumeratio: Monocotyledonae. Botanical Survey of India, Calcutta.

Kabeer, K.A.A. \& V.J. Nair 2007. Flora of Tamil Nadu - Grasses. Botanical Survey of India, Kolkata.

Kabeer, K.A.A. \& V.J. Nair 2009. Trachys copeanaA new species of Poaceae from India. Kew Bull. 62: 503-505.

Ravichandhran, P. 2013. A new species of Trachys (Poaceae) from East Coast of Tamil Nadu, India. Rheedea 23(1): 22-25.

Watson, L. \& M.J. Dallwitz 1994. The Grass Genera of the World. Second Edition. CAB International Press, Wallingford.

Received: 18.3.2017

Revised and Accepted: 4.11.2017 